\title{
Tuning the Adsorption of Aromatic Molecules on Platinum via Halogenation Rengin Peköz, ${ }^{, \dagger \dagger}$ Karen Johnston, ${ }^{\dagger, \dagger}$ and Davide Donadio*,† \\ Max Planck Institute for Polymer Research, Ackermannweg 10, 55128 Mainz, Germany, and Department of Chemical and Process Engineering, University of Strathclyde, Glasgow, United Kingdom \\ Received November 20, 2013; E-mail: pekoez@mpip-mainz.mpg.de; donadio@mpip-mainz.mpg.de
}

\begin{abstract}
The interaction of aromatic molecules with metal surfaces is of key relevance for the functionality of molecular electronics and organic electronics devices. One way to control and tune the binding properties of molecules to metals is chemical functionalization. The adsorption of halogenated benzene molecules on the (111) surface of platinum is here investigated by density functional theory calculations with non-local van der Waals correlation functional. It is found that these systems exhibit a bistable adsorption energy profile with (meta)stable chemisorption and physisorption states separated by a potential energy barrier. The relative stability of these states can be tuned by functionalizing benzene with a different number or type of halogen atoms. Our results suggest a simple rational molecular design to achieve the desired interfacial binding in organic electronic devices and in composites with interfaces between large aromatic molecules and metals.
\end{abstract}

\section{INTRODUCTION}

The interaction between aromatic molecules and metal surfaces is of fundamental importance in determining the interfacial electronic transport properties in organic thin-film devices, ${ }^{1-3}$ and the thermal and mechanical properties of self assembled monolayers ${ }^{4,5}$ and polymer composites. These interactions also affect self assembling processes on surfaces, such as the bottom-up fabrication of graphene nanoribbons, ${ }^{6}$ and are critical for the functionality of molecular switches. ${ }^{7,8}$ It is, therefore, crucial to be able to predict interfacial structures and energetics as a function of the chemical details of the adsorbed molecules and to have the possibility of rationalizing molecular design for specific applications.

Benzene is the building block unit of aromatic molecules, and benzene adsorbed on metal surfaces is one of the most widely investigated benchmark systems of organic/inorganic interfaces. ${ }^{9,10}$ Larger aromatic molecules can be produced from benzene by catalysis, and both benzene and its derivatives can be functionalized with halogen atoms. ${ }^{11,12}$ Selective halogenation may provide a simple way to modulate the interaction of aromatic molecules with metal surfaces. However, monitoring the adsorption properties of small and light molecules, such as halogenated benzene, is experimentally challenging due to the low scattering factor of light atoms in surface crystallographic methods, and to the tendency of some of these molecules to dissociate on metal surfaces. Thus, important information, such as the structure and energetics at the singlemolecule level, is still not available.

The investigation of halogenated benzene molecules on metal surfaces dates back to about a century ago, with the study of catalytic coupling of aryl via the Ullmann reaction. ${ }^{13}$ The Ullmann reaction has prompted many scanning tunneling microscopy studies of the adsorption, dissociation, and diffusion of various halo-

Max Planck Institute for Polymer Research, Ackermannweg 10, 55128 Mainz, Germany

${ }^{\dagger}$ Department of Chemical and Process Engineering, University of Strath clyde, Glasgow, United Kingdom genated benzene molecules on $\mathrm{Cu}(111) .{ }^{14-17}$ One of these studies showed that it is possible to control the amount and direction of charge transfer, and the network formation of various trisubstituted benzene molecules on $\mathrm{Cu}(111) .{ }^{17}$ The adsorption energy and configuration of larger aromatic molecules, such as pentacene on $\mathrm{Cu}(111)$, were also shown to change significantly by functionalization with fluorine atoms. ${ }^{18,19}$

In this paper, we investigate the effect of halogen substitution ( $F$, $\mathrm{Cl}$, and $\mathrm{Br}$ ) on the adsorption of benzene on $\mathrm{Pt}(111)$ by means of first-principles electronic structure calculations. Platinum is chosen as a prototype transition metal with a partially unoccupied $d$ shell and it was shown that the adsorption of conjugated systems on $d$-type transition metal surfaces is determined by an interesting combination of chemical bonding and dispersion interactions. ${ }^{20}$

Here we show that, in contrast with benzene, which is directly chemisorbed on $\operatorname{Pt}(111)$, halogenated molecules present bistable adsorption with (meta)stable chemisorption and physisorption states separated by an energy barrier. The relative stability of physisorption versus chemisorption states can be tuned by changing the number and the type of functionalizing halogen atoms. The energetics of adsorption is controlled by the structural changes of the chemisorbed molecules, the interaction between the carbon ring and the $d$-electrons of the substrate, and long-range dispersion forces. Assuming that these factors contribute nearly additively to the adsorption energy permits a detailed interpretation of the interaction of halogenated benzene molecules with $\mathrm{Pt}(111)$.

\section{METHODS}

The adsorption energy and geometry of halogenated benzene molecules on $\mathrm{Pt}(111)$ is studied using density functional theory (DFT). In these type of systems the effect of van der Waals (vdW) forces is essential and has to be explicitly taken into account to achieve a meaningful physical description and good agreement with experiments. ${ }^{21-25}$ We use the non-local vdW correlation functional, vdW-DF, ${ }^{21}$ combined with the Perdew, Burke and Ernzerhof $(\mathrm{PBE})^{26}$ local exchange. This combination of local exchange and non-local correlation functionals gives good agreement between the calculated and experimentally measured adsorption energy for benzene on $\mathrm{Au}(111) .{ }^{27}$ The core electrons were described using the PAW method ${ }^{28,29}$ and for the valence electrons a plane wave cut off of $400 \mathrm{eV}$ was used. All the calculations were performed using the VASP 4.6 code. ${ }^{30,31}$

The optimized equilibrium lattice constant of bulk Pt is $4.03 \AA$ using vdW-DF with PBE exchange, and $3.98 \AA$ using GGA-PBE, compared with the experimental value of $3.92 \AA$. The substrate is modeled as a four-layer slab of platinum atoms in a supercell with periodic boundary conditions. The two bottom layers are kept fixed at their crystallographic bulk coordinates, and the periodic images of the slabs are separated by $\sim 30 \AA$ of vacuum. To probe the effect of different coverages, calculations were performed considering the adsorption of one (halogenated) benzene molecule in two supercells with different lateral dimensions are considered, containing slabs of 16 and 30 atoms per layer of $\mathrm{Pt}$, corresponding to different coverage. The smaller cell is an orthorhombic supercell of dimensions 
$11.4 \AA \times 9.9 \AA \times 37 \AA$, equivalent to $(4 \times 4)$ hexagonal unit cells, while the larger supercell is $14.3 \AA \times 14.8 \AA \times 37 \AA$. Following the definition in Ref. [10], these systems correspond to a coverage of $\Theta=0.41$ monolayer (ML) for the smaller simulation cell and $\Theta=0.22 \mathrm{ML}$ for the larger. In the ionic relaxations the geometry optimizations were stopped when the maximum force component was less than $10 \mathrm{meV} / \AA$. The potential energy surface (PES) is calculated by fixing the $z$-coordinates of the two opposite $\mathrm{C}$ atoms attached to the halogens and adjusting this distance from the surface. All other coordinates were allowed to relax except for the fixed layers at the bottom of the Pt slab.

\section{RESULTS AND DISCUSSION}

In this section we first demonstrate the validity of the calculations by considering the benchmark system of benzene adsorption. We then systematically address the effect of the number of halogen atoms and the type of halogen atoms before presenting a detailed analysis of the adsorption trends.

\subsection{Benchmark Calculations on Adsorption of Benzene on} $\operatorname{Au}(111)$ and $\operatorname{Pt}(111)$

To validate our simulation setup and quantify the importance of dispersion interactions we computed the binding energy of benzene as a function of its distance $(z)$ from $\mathrm{Pt}$ (111) using both a standard semi-local DFT functional (PBE) ${ }^{26}$ and vdW-DF with PBE exchange. In its most favorable adsorption geometry benzene lies flat on the $\operatorname{Pt}(111)$, with its center of mass on the bridge site (B-30), ${ }^{20,32}$ where the axis formed by two opposite hydrogen atoms is rotated by $30^{\circ}$ with respect to the direction of the bridge site (Figure 1a). In this geometry we observe an adsorption minimum at a vertical distance $(z)$ of the carbon ring from the surface of 2.13 and $2.16 \AA$ for PBE and vdW-DF calculations, respectively, which is in excellent agreement with the experimental value of $2.11 \AA .{ }^{32}$ As observed from low-energy electron diffraction (LEED) experiments, ${ }^{32}$ benzene chemisorbed on $\mathrm{Pt}$ is distorted from its gas phase planar geometry, as the $\mathrm{C}-\mathrm{H}$ bonds bend upwards, due to repulsion with the underlying substrate. Bending depends on the position of $\mathrm{H}$ with respect to the atoms of the substrate. In the B-30 configuration four hydrogen atoms sit on bridge sites and bend by $0.34 \AA\left(18^{\circ}\right)$ with respect to the plane of the aromatic ring, and two sit almost on top sites and bend by $0.56 \AA\left(31^{\circ}\right)$ (Figure 1a). The adsorption energy, $E_{\mathrm{ads}}$, is defined as $E_{\text {ads }}=E_{\text {total }}-E_{\text {molecule }}-E_{\text {surface }}$ where $E_{\text {total }}, E_{\text {molecule }}$, and $E_{\text {surface }}$ are the total energies of the adsorbed system, the isolated molecule, and the clean Pt surface, respectively. $E_{\text {ads }}$ obtained using the PBE functional is $-1.19 \mathrm{eV}$, indicating that the interaction between benzene and the surface is mostly due to chemical bonding. Ihm and coworkers, ${ }^{10}$ using calorimetric measurments, observed that the binding energy of benzene on $\operatorname{Pt}(111)$ surface was coverage dependent. Using vdW-DF gives adsorption energies of $-1.61 \mathrm{eV}$ at $0.41 \mathrm{ML}$ coverage and $-1.69 \mathrm{eV}$ at the lowest coverage of $0.22 \mathrm{ML}$. Both results are within the experimental binding energy range of 1.52-1.86 eV (for $\theta=0.41 \mathrm{ML}$ ) and $1.69-2.07 \mathrm{eV}$ (for $\theta=0.22 \mathrm{ML}$ ), ${ }^{10}$ and indicate that dispersion forces have a significant role in chemisorption, augmenting the binding energy by about $0.4 \mathrm{eV}$. The potential energy surface (PES), $E_{\text {ads }}(z)$, obtained with vdW-DF (see Figure 1c) qualitatively resembles the one reported in a former theoretical work, ${ }^{20}$ and displays a very shallow minimum with $E_{\text {ads }} \sim-1 \mathrm{eV}$ at $z \sim 3.0 \AA$, due to dispersion interactions. In this marginally metastable physisorption state benzene retains its gas phase geometry (Figure 1b).

In Table 1 the adsorption energies of benzene on $\mathrm{Au}(111)$ and $\mathrm{Pt}(111)$ resulting from various state-of-the-art vdW-DF methods are compared to experiments. The comparison shows that, especially for adsorption on $\mathrm{Pt}$, the choice of the vdW correction can make a large difference. Our chosen flavor of vdW-DF(PBE) yields a good agreement with the experimental data sets.

Table 1. Adsorption energies for benzene on $\mathrm{Pt}(111)$ and $\mathrm{Au}(111)$ surfaces. On Pt the adsorption is on the B-30 site and for Au the adsorption is on the $\mathrm{H}-30$ site. The surface coverages are calculated using the definition given in Ref. [10] and correspond to $0.41 \mathrm{ML}$ for our data, for optB88-vdW and the experimental range, and $0.73 \mathrm{ML}$ for PBE+vdW ${ }^{\text {surf }}$ and DFT-D2 calculations. Energies are in $\mathrm{eV}$.

\begin{tabular}{l|cc}
\hline XC functional & $\mathrm{C}_{6} \mathrm{H}_{6} / \mathrm{Pt}(111)$ & $\mathrm{C}_{6} \mathrm{H}_{6} / \mathrm{Au}(111)$ \\
\hline PBE & 1.19 & 0.10 \\
vdW-DF (PBE) & 1.61 & 0.83 \\
vdW-DF (revPBE) & 0.92 & 0.55 \\
vdW-DF2 (PW86) & 0.86 & 0.66 \\
PBE+vdW & $1.96^{20}$ & $0.74^{20}$ \\
optB88-vdW & $2.14^{33}$ & - \\
DFT-D2 & - & $0.76^{34}$ \\
Experiment & $1.52-1.86^{10}$ & $0.64^{35}$ \\
\hline
\end{tabular}

\subsection{Adsorption of Polychlorobenzene Molecules}

Before systematically addressing the effect of halogenation, we have verified that the preferential adsorption site of halogenated benzene molecules on $\mathrm{Pt}(111)$ corresponds to that of benzene. Computing the chemisorption energy of hexafluorobenzene $\left(\mathrm{C}_{6} \mathrm{~F}_{6}\right)$ on various sites we have found that the $\mathrm{B}-30$ is the most stable one (see Section 1 in the SI). Therefore, from now on, we always consider molecules adsorbed on the B-30 site. In addition, physisorption energies are relatively insensitive to the adsorption site, as shown, for example, in the case of $1,4-\mathrm{C}_{6} \mathrm{H}_{4} \mathrm{Cl}_{2}$ on $\mathrm{Pt}(111) .{ }^{36}$

(a)
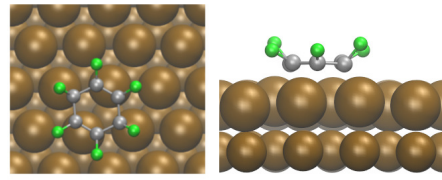

(b) 00000

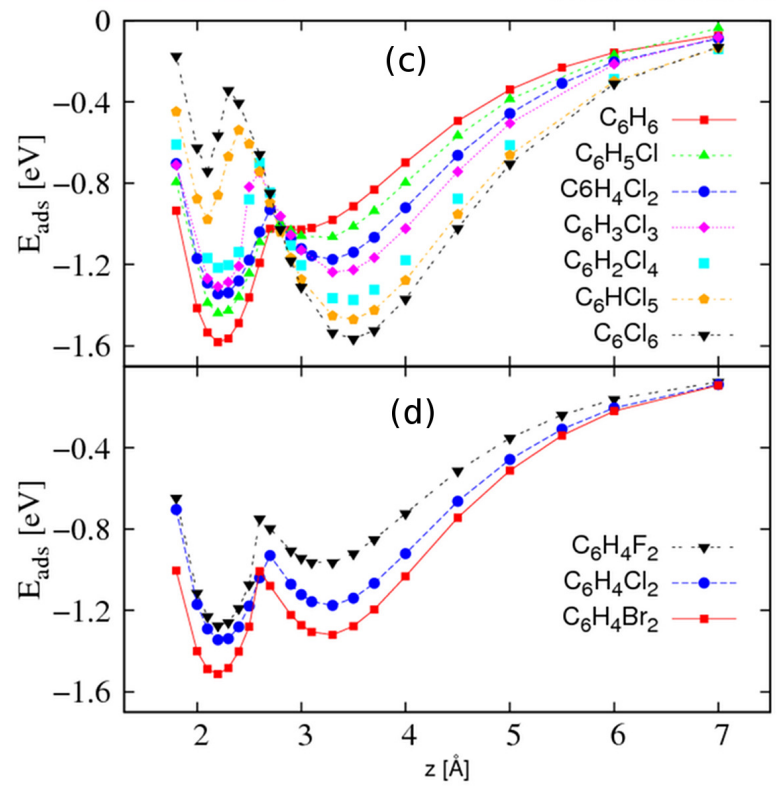

Figure 1. Relaxed geometries of benzene or halogenated benzene adsorbed at the B-30 site on $\mathrm{Pt}(111)$ for (a) chemisorbed and (b) physisorbed states. Brown, gray and green spheres represent $\mathrm{Pt}, \mathrm{C}$, and $\mathrm{H}$ or halogen atoms, respectively. Calculated adsorption energies of (c) chlorobenzenes and (d) 1,4-dihalogenated benzenes on $\mathrm{Pt}(111)$ surface at low coverage as a function of the distance between the surface plane and the constrained carbon atoms.

We proceed to examine the effect of halogenation of benzene on the PES by gradually substituting $\mathrm{H}$ atoms with $\mathrm{Cl}$ atoms. For 2,3 or 4 substitutions there are several possible configurations, so we only consider the most symmetric ones, namely, 1,4- 
$\mathrm{C}_{6} \mathrm{H}_{4} \mathrm{Cl}_{2}$ (para-dichlorobenzene), 1,3,5- $\mathrm{C}_{6} \mathrm{H}_{3} \mathrm{Cl}_{3}$ (trichlorobenzene), and 1,2,4,5- $\mathrm{C}_{6} \mathrm{H}_{2} \mathrm{Cl}_{4}$ (tetrachlorobenzene). The PESs, shown in Figure 1c, are calculated for a coverage of $0.41 \mathrm{ML}$ by fixing the coordinates of two $\mathrm{C}$ atoms and changing the distance from $\mathrm{Pt}(111)$. Each adsorption curve has two minima, one at $\sim 2.15 \AA$ and the other at 3.11-3.43 $\AA$, which correspond to chemisorbed and physisorbed states, respectively. As the number of $\mathrm{Cl}$ atoms is increased, bistability becomes more pronounced: the chemisorption minimum becomes shallower, whereas the physisorption minimum gets deeper due to the increased vdW interaction between the halogen atoms and the metallic substrate. This effect produces a crossover in the relative stability of the two minima, such that physisorption becomes energetically more favorable than chemisorption for four or more $\mathrm{Cl}$ atoms. The equilibrium distance for physisorption is also affected by halogenation, with the position of the minimum increasing from $2.97 \AA$ for benzene to $3.43 \AA$ for $\mathrm{C}_{6} \mathrm{Cl}_{6}$. An analysis of the molecular structures in the chemisorbed states allows one to understand the energetics of adsorption of chlorobenzene molecules on $\mathrm{Pt}(111)$. Like benzene, chlorobenzene molecules are deformed in the chemisorption state, while they retain their undistorted planar gas-phase geometry in the physisorption state. For instance, the adsorption geometries of $\mathrm{C}_{6} \mathrm{Cl}_{6}$ in both states are shown in Figure 1(a-b). The out-of-plane bending of $\mathrm{C}-\mathrm{H}$ and $\mathrm{C}-\mathrm{Cl}$ bonds is due to steric repulsion between $\mathrm{H}$ and $\mathrm{Cl}$ atoms and the metallic substrate, and costs a considerable amount of energy, denoted $E_{\text {bend }}$, which penalizes chemisorption. We calculate $E_{\text {bend }}$ as the difference between the single point energy of a deformed molecule in gas phase and the energy of the relaxed molecule in vacuum in the same simulation box. The $E_{\text {bend }}$ value for $\mathrm{C}_{6} \mathrm{H}_{6}$ is $1.49 \mathrm{eV}$. Bending angles are significantly larger for $\mathrm{C}-\mathrm{Cl}$ bonds $\left(22-42^{\circ}\right)$ than for $\mathrm{C}-\mathrm{H}$ bonds $\left(18-33^{\circ}\right)$ resulting in a larger average bending energy of about $0.3 \mathrm{eV}$ per bond. $E_{\text {bend }}$ is only partially compensated by the stronger attraction between chlorinated benzene and the substrate, so that the more $\mathrm{Cl}$ atoms are replaced by $\mathrm{H}$, the more energetically unfavorable the chemisorbed state. A detailed analysis of adsorption geometries and energetics is reported in Section 2 of the SI.

\subsection{Adsorption of Dihalogenated Benzene Molecules}

To probe the effect of substituting different types of halogen atoms on the chemi- and physisorption energies $\left(E_{\text {ads }}^{\text {chem }}\right.$ and $E_{\text {ads }}^{\text {phys }}$, respectively), we have computed the adsorption energy profiles of 1,4dihalogenated benzene molecules on $\mathrm{Pt}(111)$ using $\mathrm{F}, \mathrm{Cl}$ and $\mathrm{Br}$ for halogenation at a coverage of $0.41 \mathrm{ML}$, (Figure 1d). These molecules display a physisorption minimum at about $3.2 \AA$ and a deeper chemisorption minimum at $2.2 \AA$. However, the binding energies in both the chemisorbed and the physisorbed states increase (minima get deeper) as the "size" of the halogen substituents increases. In general, larger halogens are more polarizable and, therefore, have stronger vdW interactions with the substrate, thus enhancing not only their physisorption binding energy but also the contribution of dispersion interactions to the chemisorption energy.

\subsection{Adsorption of Polyhalogenated Benzene Molecules}

In order to explore the general trends and gain a better insight into the effect of halogenation on the adsorption properties of benzene on $\mathrm{Pt}(111)$, we investigated the chemisorption and physisorption of a large set of polyhalogenated benzenes with $\mathrm{F}, \mathrm{Cl}$ or $\mathrm{Br}$, where $n=1-6$ is the number of halogen atoms, and for both 0.41 and $0.22 \mathrm{ML}$ coverages. To compute the binding energies we performed a full geometry relaxation of the molecules and the top two layers of the surface. Results are plotted in Figure 2 and collected in Table 2 in SI. Depending on the type of halogen atom, the chemiand physisorption energies exhibit different behavior. For benzene functionalized with $\mathrm{Cl}$ or $\mathrm{Br}$, we observe a linear increase of the physisorption binding energy with $n$, with $\mathrm{Br}$ providing a stronger

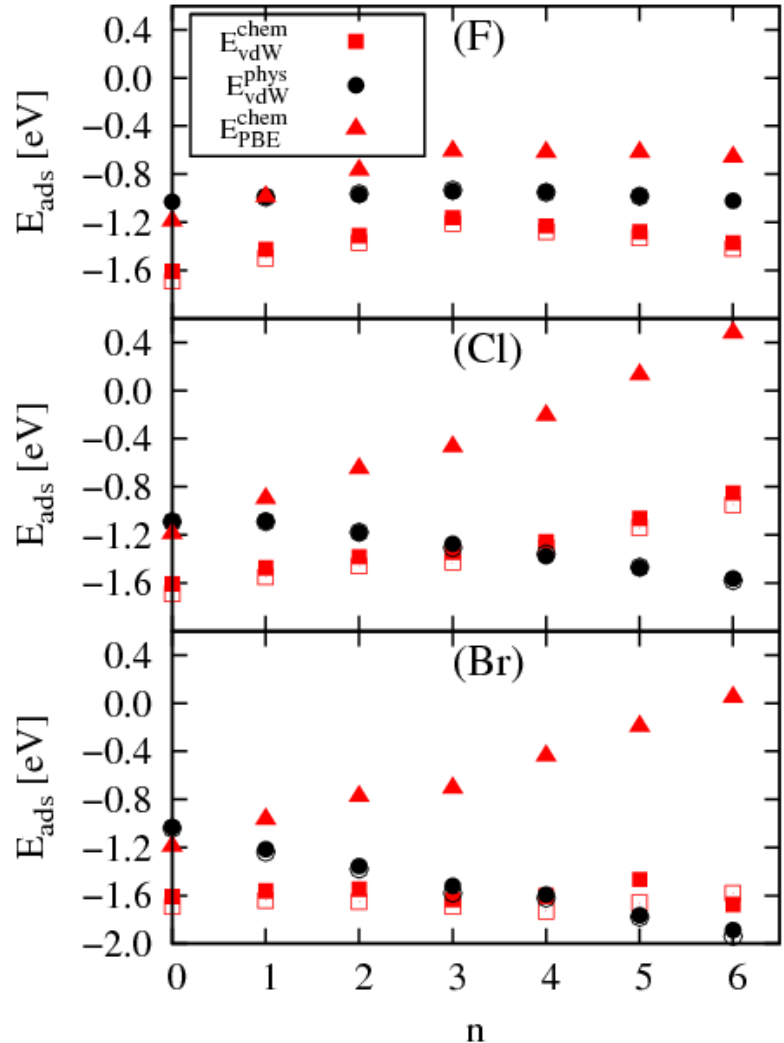

Figure 2. Calculated chemisorption and physisorption energies of polyhalogenated benzenes on $\mathrm{Pt}(111)$ surface as a function of the number of halogens using PBE and vdW-DF with $\Theta=0.22 \mathrm{ML}$ (open symbols) and 0.41ML (full symbols).

attraction than $\mathrm{Cl}$. On the other hand, $\mathrm{F}$ substitution has no sizable impact on $E_{\text {ads }}^{\text {phys }}$, which remains of the same order as the binding energy of the shallow physisorption minimum observed for benzene. As in the cases discussed above, the physisorbed molecules retain their gas-phase planar geometry, and we do not observe significant variations in the electronic structure of either the molecules or the surface. Therefore, these trends in $E_{\text {ads }}^{\text {phys }}$ simply stem from the enhanced vdW interaction between the halogen atoms and the surface as a function of the polarizability of the halogens, which is larger than that of hydrogen. Decreasing the coverage from 0.41 to $0.22 \mathrm{ML}$ systematically lowers $E_{\text {ads }}^{\text {phys }}$ by $0.04 \mathrm{eV}$, indicating a weak repulsive lateral interaction between the adsorbate.

Trends in chemisorption energies are very different, depending on the type of halogen atoms used for substitution (Figure 2). As shown in Figure 1a, substituting $\mathrm{H}$ with $\mathrm{Cl}$ weakens chemisorption and strengthens physisorption, so that physisorption binding energy increases monotonically and a crossover between $E_{\text {ads }}^{\text {phys }}$ and $E_{\text {ads }}^{\text {chem }}$ occurs between 3 and $4 \mathrm{Cl}$ atoms. Substituting $\mathrm{H}$ with $\mathrm{Br}$ does not produce large variations in $E_{\mathrm{ads}}^{\mathrm{chem}}$, which remains similar to the binding energy of benzene, $\sim-1.6 \mathrm{eV}$. Also bromination gives rise to a crossover between $E_{\text {ads }}^{\text {phys }}$ and $E_{\text {ads }}^{\text {chem }}$ between 4 and $5 \mathrm{Br}$ atoms, due to the monotonic increase of the physisorption binding energy with $n .^{*}$ On the other hand, the binding energy of chemisorbed polyfluorobenzene is non-monotonic: it decreases as $n$ goes from 1 to 3 , and increases again as $n$ goes from 3 to 6 : no crossover with physisorption is observed.

${ }^{*}$ We note that chlorinated and brominated benzenes with $n=6$ tend to dissociate easily when they are brought in close contact with $\mathrm{Pt}$, therefore the results in Figure 2 for large $n$ may correspond to marginally stable structures, most likely very difficult to stabilize experimentally. 
Table 2. Buckling energy of the surface, in eV, and the average outward displacement, in $\AA$, of the surface Pt atoms bounded to the molecules with respect to the unbound Pt atoms. The calculated $E_{\text {buck }}$ and $d_{\text {buck }}$ for benzene is $0.36 \mathrm{eV}$ and $0.14 \AA$, respectively.

\begin{tabular}{c|rr|rr|rr|rr|rr|rrr}
\hline & \multicolumn{9}{|c|}{$\Theta=0.41$} & \multicolumn{3}{c|}{$\mathrm{C}$} & \multicolumn{3}{c|}{$\mathrm{F}$} & \multicolumn{2}{c|}{$\mathrm{Cl}$} & \multicolumn{3}{c}{$\mathrm{Br}$} \\
& \multicolumn{3}{|c|}{$\mathrm{F}$} & \multicolumn{2}{c|}{$\mathrm{Cl}$} & \multicolumn{2}{c|}{$\mathrm{Br}$} \\
$n$ & $d_{\text {buck }}$ & $E_{\text {buck }}$ & $d_{\text {buck }}$ & $E_{\text {buck }}$ & $d_{\text {buck }}$ & $E_{\text {buck }}$ & $d_{\text {buck }}$ & $E_{\text {buck }}$ & $d_{\text {buck }}$ & $E_{\text {buck }}$ & $d_{\text {buck }}$ & $E_{\text {buck }}$ \\
\hline 1 & 0.15 & 0.38 & 0.15 & 0.38 & 0.12 & 0.42 & 0.13 & 0.42 & 0.13 & 0.42 & 0.12 & 0.42 \\
2 & 0.15 & 0.40 & 0.15 & 0.40 & 0.12 & 0.44 & 0.14 & 0.43 & 0.14 & 0.44 & 0.12 & 0.44 \\
3 & 0.16 & 0.44 & 0.16 & 0.43 & 0.13 & 0.47 & 0.14 & 0.48 & 0.14 & 0.47 & 0.13 & 0.46 \\
4 & 0.17 & 0.45 & 0.17 & 0.43 & 0.13 & 0.46 & 0.15 & 0.49 & 0.15 & 0.46 & 0.13 & 0.46 \\
5 & 0.18 & 0.46 & 0.18 & 0.44 & 0.13 & 0.48 & 0.16 & 0.49 & 0.15 & 0.47 & 0.13 & 0.48 \\
6 & 0.19 & 0.45 & 0.19 & 0.46 & 0.13 & 0.49 & 0.17 & 0.52 & 0.17 & 0.50 & 0.15 & 0.52 \\
\hline
\end{tabular}

To achieve a consistent interpretation of the observed trends in chemisorption energies, one has to identify the main contributions to $E_{\text {ads }}^{\mathrm{chem}}$, the largest of which is attractive and corresponds to the chemical bonding between the aromatic carbon ring and the $\mathrm{Pt}$ surface. This term is augmented by the vdW interactions of the molecules with the surface. It is possible to single out the vdW contribution by comparing vdW-DF binding energies to those obtained with the PBE functional. Such comparison shows that local electronic interactions are predominantly responsible for the nonmonotonic trend of the $E_{\text {ads }}^{\mathrm{chem}}$ of polyfluorobenzene, since PBE calculations give nearly the same chemisorption energy for $n=3-6$, as shown in Figure 2. We also note that dispersion forces contribute significantly to stabilize the chemisorbed state of chlorinated and brominated benzene molecules. In general, the vdW contribution is linearly proportional to the number of substituted halogens as demonstrated in Figure 3 and further details are presented in Section 3 in SI.

As mentioned above, benzene and halogenated benzene molecules in the chemisorbed states are deformed, with $\mathrm{C}-\mathrm{H}$ and $\mathrm{C}-X(X=\mathrm{F}, \mathrm{Cl}$, or $\mathrm{Br})$ bonds bent away from the surface, due to steric repulsion. The bending energy penalizes chemisorption and ranges from $1.49 \mathrm{eV}$ for benzene to $3.67 \mathrm{eV}$ for $\mathrm{C}_{6} \mathrm{~F}_{6}$. $E_{\text {bend }}$ increases monotonically with the number of halogen substituents, and spans roughly the same energy range regardless of their type (Figure 4). In the case of $\mathrm{Cl}$ and $\mathrm{Br}$ substituents, $E_{\text {bend }}$ is fairly linear as a function of $n$, whereas it deviates significantly from linearity for fluorinated benzene. Deviations from linearity depend on the position of the substituting halogen atoms with respect to the substrate and on collective interactions within the deformed molecule. In particular, the discontinuity from $n=2$ to $n=3$ is due to the presence of one halogen atom on top of a Pt atom.

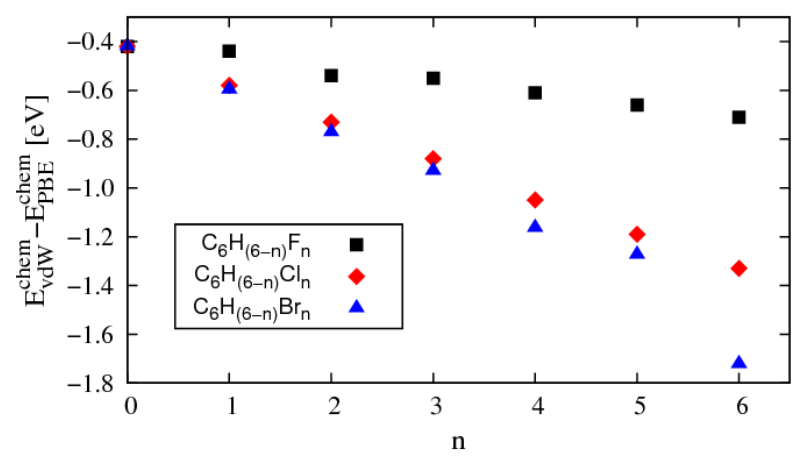

Figure 3. Contribution of dispersion interactions to the chemisorption energies of polyhalogenated benzenes with coverage $\Theta=0.41 \mathrm{ML}$, computed as the difference between vdW-DF and PBE adsorption energies.

In addition, we observe buckling of the surface Pt atoms in contact with the chemisorbed molecules. Buckling displacements can be as large as $0.14 \AA$ for benzene, up to $0.19 \AA$ for hexahalogenated benzene molecules. The energy required for the buckling of the sur- face atoms ranges from $0.36 \mathrm{eV}$ for benzene to $0.48 \mathrm{eV}$ for $\mathrm{C}_{6} \mathrm{HBr}_{5}$. Buckling energies and displacements for each system with $\theta=0.41$ and $\theta=0.22 \mathrm{ML}$ are reported in Table 2 .

Calculations of the adsorption energy at different coverage show that the interaction between chemisorbed molecules is weakly repulsive. Reducing coverage from 0.41 to $0.22 \mathrm{ML}$ leads to a systematic negative shift of $E_{\text {ads }}^{\text {chem }}$ of less than $0.1 \mathrm{eV}$. An exception is the case of $\mathrm{C}_{6} \mathrm{HBr}_{5}$ where the difference in $E_{\text {ads }}^{\text {chem }}$ is $0.2 \mathrm{eV}$. Further decreasing the coverage to $0.14 \mathrm{ML}$ decreases the $E_{\text {ads }}^{\text {chem }}$ of $\mathrm{C}_{6} \mathrm{HBr}_{5}$ by $0.1 \mathrm{eV}$. These results indicate that, although they are important for determining the absolute binding energies, surface buckling and intermolecular interactions have a much smaller impact on the trends of $E_{\mathrm{ads}}^{\mathrm{chem}}$ than chemical interactions, dispersion forces and molecular deformations. We also estimate the contribution from dipole-image interaction ${ }^{37}$ to the chemisorption energy, which is about $0.02 \mathrm{eV}$ for the molecules with the largest dipole moment, i.e. fluorobenzenes. Thus, we conclude that electrostatics has a negligible impact on the chemisorption energy.

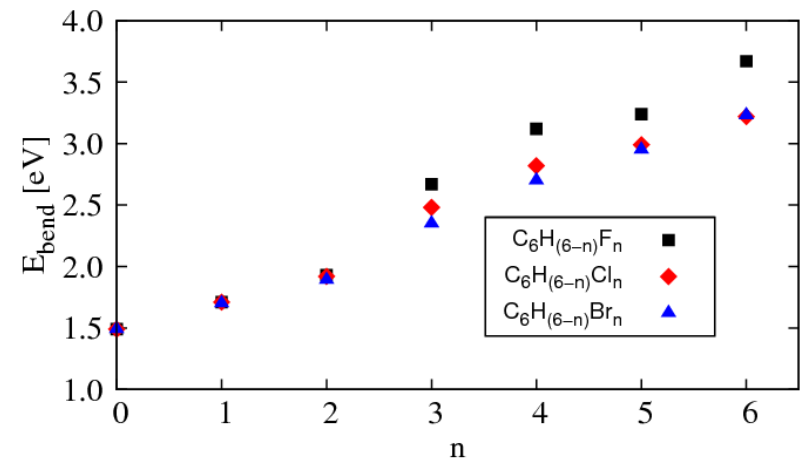

Figure 4. Bending energy of polyhalogenated benzene on $\operatorname{Pt}(111)$ the number of halogen substitution. Deformation energies are independent on coverage.

The combination of the effects listed above produces nearly linear trends in the chemisorption energy of chlorinated and brominated benzene molecules as a function of $n$. This behavior allows us to define a contribution to $\Delta \varepsilon_{\text {ads }}^{\mathrm{X}}$ per halogen atom $X=\mathrm{Cl}$ or $\mathrm{Br}$, as:

$$
\Delta \varepsilon_{a d s}^{\mathrm{X}}=\left(E_{\mathrm{ads}}\left[\mathrm{C}_{6} \mathrm{H}_{6-\mathrm{n}} \mathrm{X}_{\mathrm{n}}\right]-E_{\mathrm{ads}}\left[\mathrm{C}_{6} \mathrm{H}_{6}\right]\right) / n
$$

which can be calculated for any chosen $\mathrm{C}_{6} \mathrm{H}_{6-n} \mathrm{X}_{n}$ molecule or averaged over a set of them. The total chemisorption energy for polychlorinated and polybrominated benzene on $\mathrm{Pt}(111) \mathrm{can}$, therefore, be expressed as $E_{\text {ads }}^{\text {chem }}\left[\mathrm{C}_{6} \mathrm{H}_{6-\mathrm{n}} \mathrm{X}_{\mathrm{n}}\right]=E_{\text {ads }}^{\text {chem }}\left[\mathrm{C}_{6} \mathrm{H}_{6}\right]+n \Delta \varepsilon_{\text {ads }}^{\mathrm{X}}$. Using $\mathrm{C}_{6} \mathrm{Cl}_{6}$ and $\mathrm{C}_{6} \mathrm{Br}_{6}$ to calculate $\Delta \varepsilon_{\text {ads }}^{\mathrm{X}}$, the adsorption energies of the other polychlorinated and polybrominated molecules can be retrieved with an accuracy within $0.1 \mathrm{eV}$. On the other hand, in the case of fluorination a linear fit is not suitable to describe chemisorption, because of the non-monotonic trend of $E_{\mathrm{ads}}^{\mathrm{chem}}$. This fit may eventually serve as a simple model to predict how $E_{\text {ads }}^{\text {chem }}$ would 
change when larger aromatic molecules are halogenated, or in case of mixed halogenation. ${ }^{18,19}$

\section{CONCLUSIONS}

In summary, using first-principles calculations including van der Waals interactions, we have shown that the adsorption of polyhalogenated benzene on the $\operatorname{Pt}(111)$ surface displays a bistable adsorption energy profile, with a chemisorption and a physisorption state, which differ in their bonding properties, equilibrium distances, and electronic structures. By changing the number or the type of halogen atoms it is possible to systematically control the relative stability of chemisorption and physisorption states. The various types of interactions determining the binding energy between halogenated molecules and the metallic substrate have been evaluated and ranked. For chlorinated and brominated benzenes these contributions lead to simple linear trends in both chemisorption and physisorption energies, which make it relatively easy to predict the binding properties of a set of halogenated molecules to surfaces by knowing the binding energy of a smaller subset. These results suggest that selective halogenation of phenyl rings can be exploited as a tool for molecular design to control the nature of surface bonding of large aromatic molecules or polymer side-chains to metal surfaces. Such tools offer broad perspectives of exploitation in all those applications, where the interaction between molecules and metallic surfaces is critical, such as polymer composites, surfaceassisted molecular assembly, organic electronic devices, molecular junctions and molecular switches.

Acknowledgement The authors thank L. M. Ghiringhelli and E. McNellis for valuable discussions and suggestions, and C. Mangold for a critical reading of the manuscript. We acknowledge the RZG of the Max Planck Society for computational resources. R.P. and D.D. were funded by the MPRG program of the MPG. Funding for K.J. was provided by the German Research Foundation under the Priority Program DFG SPP 1369.

Supporting Information Available: Physi- and chemisorption energies and geometrical details of the systems. Relaxed geometries of polychlorobenzene. This material is available free of charge via the Internet at http://pubs.acs.org/.

\section{References}

(1) Cicoira, F.; Santato, C. Organic light emitting field effect transistors: advances and perspectives. Adv. Funct. Mater. 2007, 17, 3421-3434.

(2) Kronik, L.; Koch, N. Electronic Properties of Organic-Based Interfaces. MRS Bull. 2010, 35, 417-421.

(3) Tseng, T.-C. et al. Charge-transfer-induced structural rearrangements at both sides of organic/metal interfaces. Nature Chem. 2010, 2, 374-379.

(4) Ong, W.-L.; Rupich, S. M.; Talapin, D. V.; McGaughey, A. J. H.; Malen, J. A. Surface chemistry mediates thermal transport in threedimensional nanocrystal arrays. Nature Mater. 2013, 12, 410-415.

(5) Losego, M. D.; Grady, M. E.; Sottos, N. R.; Cahill, D. G.; Braun, P. V. Effects of chemical bonding on heat transport across interfaces. Nature Mater. 2012, 11, 502-506.

(6) Cai, J.; Ruffieux, P.; Jaafar, R.; Bieri, M.; Braun, T.; Blankenburg, S.; Muoth, M.; Seitsonen, A. P.; Saleh, M.; Feng, X.; Müllen, K.; Fasel, R. Atomically precise bottom-up fabrication of graphene nanoribbons. Nature 2010, 466, 470-473.

(7) Li Huang, Y.; Lu, Y.; Niu, T. C.; Huang, H.; Kera, S.; Ueno, N.; Wee, A. T. S.; Chen, W. Reversible Single-Molecule Switching in an Ordered Monolayer Molecular Dipole Array. Small 2012, 8, 1423-1428

(8) Liu, W.; Filimonov, S. N.; Carrasco, J.; Tkatchenko, A. Molecular switches from benzene derivatives adsorbed on metal surfaces. Nature Comm. 2013, 4, 2569.

(9) Held, G.; Braun, W.; Steinrück, H.-P.; Yamagishi, S.; Jenkins, S. J.; King, D. A. Light-Atom Location in Adsorbed Benzene by Experiment and Theory. Phys. Rev. Lett. 2001, 87, 216102.

(10) Ihm, H.; Ajo, H. M.; Gottfried, J. M.; Bera, P.; Campbell, C. T. Calorimetric Measurement of the Heat of Adsorption of Benzene on Pt(111). J. Phys. Chem. B 2004, 108, 14627-14633.

(11) Ratnasamy, P.; Singh, A. P.; Sharma, S. Halogenation over zeolite catalysts. Appl. Catal. A 1996, 135, 25-55.

(12) Watson, M. D.; Fechtenkötter, A.; Müllen, K. Big Is Beautiful Aromaticity Revisited from the Viewpoint of Macromolecular and Supramolecular Benzene Chemistry. Chem. Rev 2001, 101, 1267-1300.

(13) Ullman, F.; Bielecki, J. Ueber Synthesen in der Biphenylreihe. Ber. Dtsch. Chem. Ges. 1901, 34, 2174-2185.
(14) Weiss, P. S.; Kamna, M. M.; Graham, T. M.; Stranick, S. J. Imaging Benzene Molecules and Phenyl Radicals on $\mathrm{Cu}(111)$. Langmuir 1998, 14, $1284-1289$.

(15) Hla, S. W.; Kühnle, A.; Bartels, L.; Meyer, G.; Rieder, K. H. Controlled lateral manipulation of single diiodobenzene molecules on the $\mathrm{Cu}(111)$ surface with the tip of a scanning tunnelling microscope. Surf. Sci. 2000, 454-456, 1079-1084.

(16) McCarty, G. S.; Weiss, P. S. Footprints of a Surface Chemical Reaction Dissociative Chemisorption of p-Diiodobenzene on $\mathrm{Cu}(111)$. J. Phys. Chem. B 2002, 106, 8005-8008

(17) Simpson, S.; Zurek, E. Substituted Benzene Derivatives on the $\mathrm{Cu}(111)$ Surface. J. Phys. Chem. C 2012, 116, 12636-12643.

(18) Koch, N.; Gerlach, A.; Duhm, S.; Glowatzki, H.; Heimel, G.; Vollmer, A. Sakamoto, Y.; Suzuki, T.; Zegenhagen, J.; Rabe, J. P.; Schreiber, F. Adsorption-Induced Intramolecular Dipole: Correlating Molecular Conformation and Interface Electronic Structure. J. Am. Chem. Soc. 2008, 130, $7300-7304$.

(19) Shi, X.-Q.; Li, Y.; Van Hove, M. A.; Zhang, R.-Q. Interactions between Organics and Metal Surfaces in the Intermediate Regime between Physisorption and Chemisorption. J. Phys. Chem. C 2012, 116, 23603-23607.

(20) Liu, W.; Carrasco, J.; Santra, B.; Michaelides, A.; Scheffler, M. Tkatchenko, A. Benzene adsorbed on metals: Corcerted effect of covalency and van der Waals bonding. Phys. Rev. B 2012, 86, 245405.

(21) Dion, M.; Rydberg, H.; Schröder, E.; Langreth, D. C.; Lundqvist, B. I. Van der Waals Density Functional for General Geometries. Phys. Rev. Lett. 2004, 92, 246401

(22) Tkatchenko, A.; Scheffler, M. Accurate Molecular Van Der Waals Interactions from Ground-State Electron Density and Free-Atom Reference Data. Phys. Rev. Lett. 2009, 102, 073005.

(23) Ruiz, V. G.; Liu, W.; Zojer, E.; Scheffler, M.; Tkatchenko, A. DensityFunctional Theory with Screened van der Waals Interactions for the Modeling of Hybrid Inorganic-Organic Systems. Phys. Rev. Lett. 2012, 108, 146103.

(24) Atodiresei, N.; Caciuc, V.; Lazić, P.; Blügel, S. Chemical versus van der Waals Interaction: The Role of the Heteroatom in the Flat Absorption of Aromatic Molecules $\mathrm{C} 6 \mathrm{H} 6, \mathrm{C} 5 \mathrm{NH} 5$, and $\mathrm{C} 4 \mathrm{~N} 2 \mathrm{H} 4$ on the $\mathrm{Cu}(110)$ Surface. Phys. Rev. Lett. 2009, 102, 136809.

(25) Grimme, S.; Antony, J.; Ehrlich, S.; Krieg, H. A consistent and accurate ab initio parametrization of density functional dispersion correction (DFT-D) for the 94 elements H-Pu. J. Chem. Phys. 2010, 132, 154104.

(26) Perdew, J. P.; Burke, K.; Ernzerhof, M. Generalized Gradient Approximation Made Simple. Phys. Rev. Lett. 1996, 77, 3865-3868.

(27) Johnston, K.; Harmandaris, V. Properties of Benzene Confined between Two Au(111) Surfaces Using a Combined Density Functional Theory and Classical Molecular Dynamics Approach. J. Phys. Chem. C 2011, 115 $14707-14717$.

(28) Blöchl, P. E. Projector augmented-wave method. Phys. Rev. B 1994, 50, 17953.

(29) Kresse, G.; Joubert, D. From ultrasoft pseudopotentials to the projector augmented-wave method. Phys. Rev. B 1999, 59, 1758.

(30) Kresse, G.; Furthmüller, J. Efficiency of ab-initio total energy calculations for metals and semiconductors using a plane-wave basis set. Comput. Mater. Sci. 1996, 6, 15-50.

(31) Kresse, G.; Furthmüller, J. Efficient iterative schemes for ab initio totalenergy calculations using a plane-wave basis set. Phys. Rev. B 1996, 54, 11169 .

(32) Wander, A.; Held, G.; Hwang, R. Q.; Blackman, G. S.; Xu, M. L.; de Andres, P.; Van Hove, M. A.; Somorjai, G. A. A diffuse LEED study of the adsorption structure of disordered benzene on $\operatorname{Pt}(111)$. Surf. Sci. 1991, $249,21-34$

(33) Zhang, R.; Hensley, A. J.; McEwen, J.-S.; Wickert, S.; Darlatt, E.; Fischer, K.; Schöppke, M.; Denecke, R.; Streber, R.; Lorenz, M.; Papp, C.; Steinrük, H.-P. Integrated X-ray photoelectron spectroscopy and DFT characterization of benzene adsorption on $\operatorname{Pt}(111), \operatorname{Pt}(355)$ and $\operatorname{Pt}(322)$ surfaces. Phys. Chem. Chem. Phys. 2013, 15, 20662-20671.

(34) Tonigold, K.; Gross, A. Adsorption of small aromatic molecules on the (111) surfaces of noble metals: A density functional theory study with semiempirical corrections for dispersion effects. J. Chem. Phys. 2010, 132, 224701 .

(35) Syomin, D.; Kim, J.; Koel, B. E.; Ellison, G. B. Identification of Adsorbed Phenyl $\left(\mathrm{C}_{6} \mathrm{H}_{5}\right)$ Groups on Metal Surfaces: Electron-Induced Dissociation of Benzene on Au(111). J. Phys. Chem. B 2001, 105, 8387.

(36) Peköz, R.; Johnston, K.; Donadio, D. Adsorption of dichlorobenzene on $\mathrm{Au}$ and Pt stepped surfaces using van der Waals density functional theory. J. Phys. Chem. C 2012, 116, 20409.

(37) Maschhoff, B. L.; Cowin, J. P. Corrected electrostatic model for dipoles adsorbed on a metal surface. J. Chem. Phys. 1994, 101, 8138-8151. 
Graphical TOC Entry

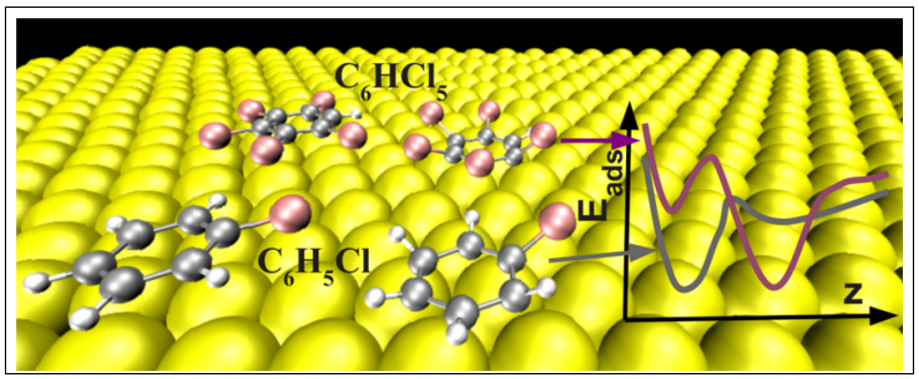

